\title{
Ferulic Acid: A Novel Inducer of Antioxidant Enzymes in Wheat (Triticum aestivum L.) Seedlings
}

\author{
A. Sharma, R.D. Bhardwat* and A.K. Gupta \\ Department of Biochemistry, Punjab Agricultural University, Ludhiana-141004, Punjab, India \\ (Received 16 June 2014; Accepted 29 September 2014; \\ Communicated by A. Pécsváradi)
}

\begin{abstract}
The present study reports the effects of pre-treatment with ferulic acid (FA) on antioxidant response of wheat seedlings. In comparison to hydropriming, 100 and $150 \mathrm{ppm}$ of FA significantly enhanced seedling growth of wheat at 6th day after germination (DAG). However, $1000 \mathrm{ppm}$ of FA led to reduction in seedling growth. Roots and shoots of wheat seedlings pre-treated with $100 \mathrm{ppm}$ of FA showed significant upregulation of peroxidase (POX), ascorbate peroxidase (APX) activities. Although catalase (CAT) remained unaffected in the roots, but showed about 2-fold increase in the shoots. Despite of low glutathione reductase (GR) and high polyphenol oxidase (PPO) activities in the shoots and roots, respectively, ascorbic acid and total phenolic contents also increased at 6th DAG which may be due to the activation of their biosynthetic pathways in seedlings pre-treated with $100 \mathrm{ppm}$ of FA. Proline content of wheat seedlings pre-treated with $100 \mathrm{ppm}$ of FA remained unaffected. Results signify the role of FA pre-treatment in augmenting the antioxidant response of wheat and thereby suggest that at lower concentrations, it can be used for improving performance of wheat under various environmental constraints.
\end{abstract}

Keywords: antioxidant enzymes, ferulic acid, wheat

\section{Introduction}

Induction of the antioxidant defense system is crucial for imparting resistance against various abiotic stresses. These abiotic stresses viz. drought, heat, salinity, light etc. cause accumulation of reactive oxygen species (ROS) such as superoxide radicals $\left(\cdot \mathrm{O}_{2}^{-}\right)$, hydrogen peroxide $\left(\mathrm{H}_{2} \mathrm{O}_{2}\right)$ and hydroxyl radicals $\left(\cdot \mathrm{OH}^{-}\right)$thereby creating a state of oxidative stress (Foyer and Noctor 2013). The response of a cultivar to tolerate any kind of abiotic stress is related with the higher potential of detoxifying enzymes to tackle the stress. Various enzymes involved in scavenging ROS are superoxide dismutase (SOD), ascorbate peroxidase (APX), glutathione reductase (GR), catalase (CAT) and peroxidase (POX) whereas non-enzymatic antioxidants include glutathione, ascorbic acid, phenols, proline and carotenoids (Sharma et al. 2012). Upregulation of SOD and CAT activities was correlated with drought tolerance of wheat (Devi et al. 2012). Therefore the impor-

\footnotetext{
* Corresponding author; E-mail: rachana1981_gautam@pau.edu
} 
tance lies in increasing the endogenous antioxidants, which can alleviate the detrimental effects of elevated ROS under stressed conditions.

Salicylic acid (SA) is a known inducer of antioxidant enzymes (Ghafiyehsanj et al. 2013; Kang et al. 2013). However, very little information is available on additional phenolic compounds such as ferulic acid (FA) which has higher antioxidant potential. Ferulic acid (3-methoxy-4-hydroxycinnamic acid) is the major phenolic acid occurring in the cell wall of monocots. Guo and Beta (2013) reported that FA is a major phenolic acid in whole grain cereals. Structurally, FA has an electron donating group on benzene ring (3-methoxy and 4-hydroxyl) that provides it an additional property of terminating free radical chain reaction Teixeira et al. (2013). Srinivasan et al. (2007) showed that ability of FA to act as a strong antioxidant is due to its phenolic nucleus and extended side chain conjugation. Most of the earlier studies involved spraying the plants with salicylic acid in order to activate the antioxidant defense system (Appu and Muthukrishnan 2014). However, seed priming seems to be more promising technique to produce tolerant plants against various stresses (Chen and Arora 2013). Moreover, it is highly economic and less labour intensive. Such studies are lacking in wheat which is the second most important cereal crop in the world. Therefore in this study, we report FA as an inducer of antioxidant system in wheat.

\section{Materials and Methods}

Wheat (PBW 621) seeds were pre-treated with water (control) as well as varying concentrations of FA for 14 hours and dried in the incubator at $25 \pm 2{ }^{\circ} \mathrm{C}$. Pre-treated seeds were germinated in plastic cups $\left(250 \mathrm{~cm}^{3}\right)$ filled with $220 \mathrm{~cm}^{3}$ of its volume containing untreated and well-irrigated soil having $\mathrm{pH} 8.0$, electrical conductivity of $0.12 \mathrm{mmhos} \mathrm{cm} \mathrm{cm}^{-1}$ and organic carbon content of $0.51 \%$. Lengths of roots and shoots and biomass of roots, shoots and endosperms were determined on 6th day after germination (DAG). Dry weight of different tissues was determined after drying the tissues at $60^{\circ} \mathrm{C}$ till the constant weight was obtained.

\section{Extraction and assays of enzymatic and non-enzymatic antioxidants}

Activities of antioxidant enzymes and contents of non-enzymatic antioxidants were determined in the roots and shoots of wheat seedlings at 6th DAG. Enzymes were extracted at $4{ }^{\circ} \mathrm{C}$ and assayed at $25^{\circ} \mathrm{C}$.

Catalase was extracted with $50 \mathrm{mM}$ sodium phosphate buffer $(\mathrm{pH} 7.5)$ containing $1 \%$ polyvinyl pyrrolidone (PVP). The extraction buffer for APX was similar to that of CAT except that additionally it contained $1 \mathrm{mM}$ ascorbate. Catalase (EC 1.11.1.6) and APX (EC 1.11.1.1) were assayed according to the methods earlier standardized in the lab (Devi et al. 2012). Glutathione reductase, POX and SOD were extracted and estimated by the methods earlier described (Devi et al. 2012). Polyphenol oxidase (PPO, EC 1.14.18.1) was extracted from $200 \mathrm{mg}$ each of roots and shoots with $2 \mathrm{ml}$ of $100 \mathrm{mM}$ sodium phosphate buffer ( $\mathrm{pH}$ 6.8) centrifuged at $10000 \times g$ (Zauberman et al. 1991). Reaction mixture 
contained $100 \mathrm{mM}$ sodium phosphate buffer (pH 6.8), $100 \mathrm{mM}$ 4-methyl catechol and enzyme extract. Absorbance at $420 \mathrm{~nm}$ was recorded at an interval of $1 \mathrm{~min}$ up to $3 \mathrm{~min}$. One unit of enzyme activity was defined 0.01 increase in absorbance. Protein content was estimated using folin phenol's reagent (Lowry et al. 1951).

The ascorbate content was determined by the method described by Chugh et al. (2011). The content of total phenols was determined by the method of Swain and Hills (1959). The proline content in roots and shoots was determined by adopting methods of Bates et al. (1973). Hydrogen peroxide content was determined according to the method described by Alexieva et al. (2001).

\section{Statistical analysis}

Growth data has been presented as mean \pm SD of three samples of 18 seedlings each. It was statistically analyzed by applying one-way analysis of variance (ANOVA) followed by post-hoc analysis, the LSD (least significant difference) test. Data for other biochemical parameters was analysed by using Student's $t$-test.

\section{Results}

\section{Effect of FA pre-treatment on seedling growth}

In comparison to hydroprimed seedlings, pre-treatment with $100 \mathrm{ppm}$ of FA promoted seedling growth of wheat (Table 1). Pre-treatment with 100 ppm of FA enhanced root and shoot lengths by about $12 \%$ whereas 150 ppm caused an increase of more than $10 \%$. Fresh weights of shoots increased after exogenous application of 100 and $150 \mathrm{ppm}$ of FA whereas dry weights remained unaffected (Table 1). However, 1000 ppm of FA decreased lengths as well as biomass of roots and shoots. Shoot lengths and fresh biomass of seedlings pre-treated with 1000 ppm of FA decreased by 20 and $12 \%$, respectively (Table 1). However, dry weights of endosperms increased in seedlings treated with $1000 \mathrm{ppm}$ of FA (Table 1). Based upon seedling growth, 100 ppm of FA was chosen for studying the effect of FA pre-treatment on antioxidant defense system.

\section{Influence of FA pre-treatment on antioxidative enzymes and $\mathrm{H}_{2} \mathrm{O}_{2}$ content}

In comparison to hydroprimed seedlings, SOD activity remained unchanged in FA-treated seedlings (Table 2). In comparison to water-primed seedlings, although CAT activity of roots of wheat seedlings pre-treated with 100 ppm of FA remained unaffected but it increased by about 2-fold in shoots (Table 2). Peroxidase activity in FA-treated seedlings showed an elevation of about 3.5-fold in the roots and 1.7-fold in the shoots (Table 2). In comparison to hydro-primed seedlings, $100 \mathrm{ppm}$ of FA-upregulated APX activity of roots by about 2-fold whereas shoots showed an increase of 1.3-fold (Table 2). Glutathione reductase activity remained unaffected in the roots of wheat seedlings pre-treated with $100 \mathrm{ppm}$ of FA but downregulated in the shoots. Hydrogen peroxide content decreased by 


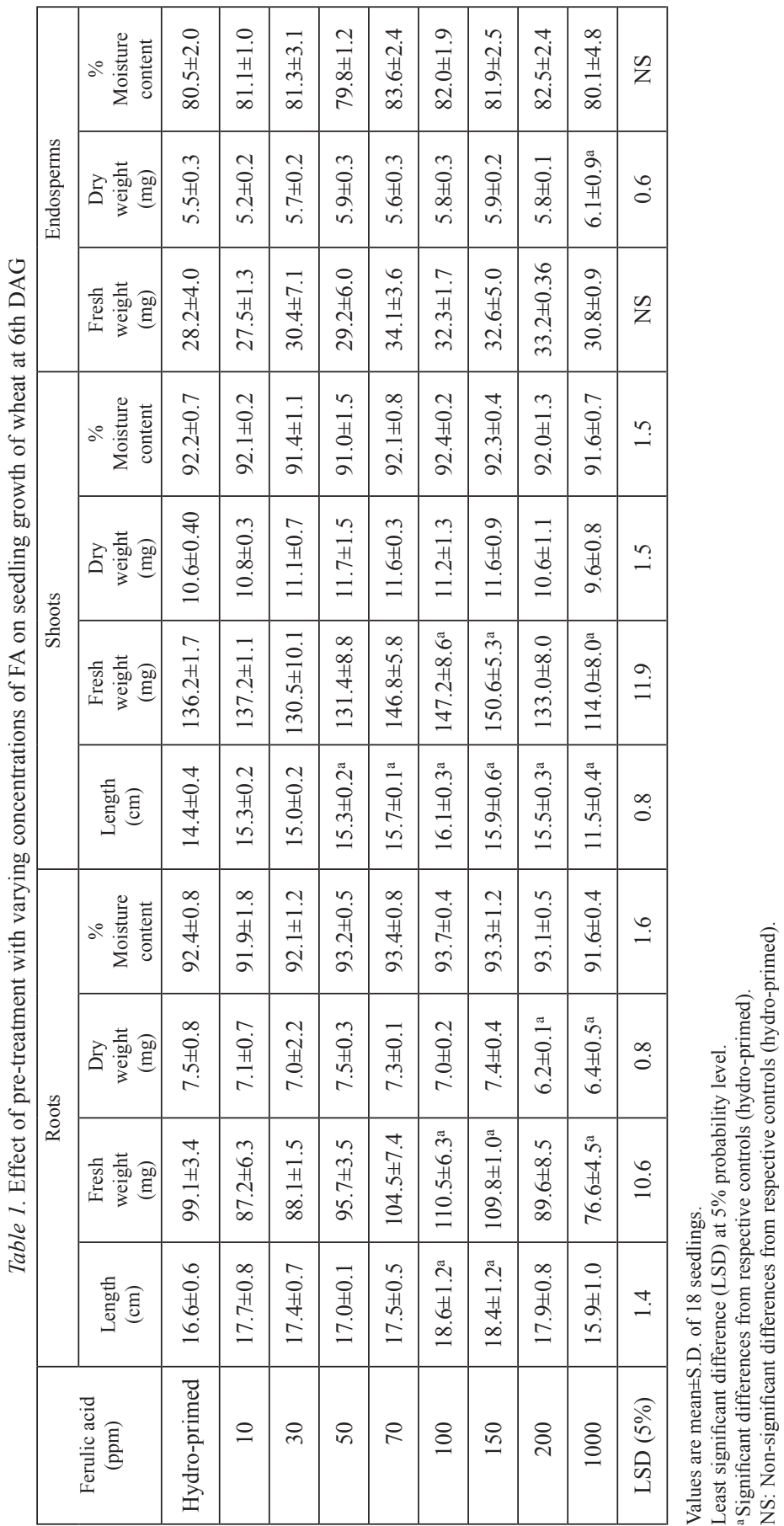




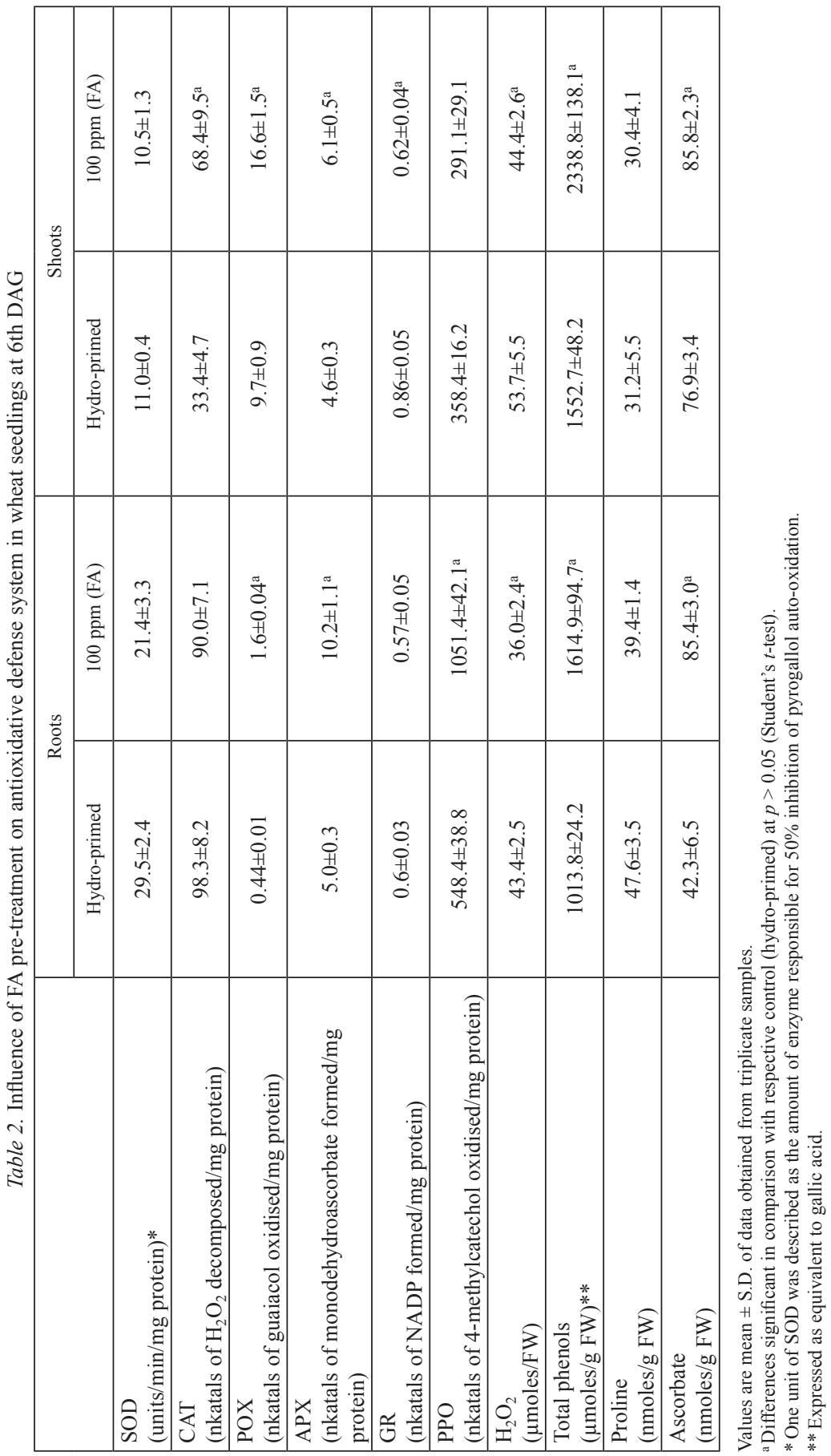


about $17 \%$ in roots and shoots of wheat seedlings pre-treated with $100 \mathrm{ppm}$ of FA. Pretreatment with 100 ppm of FA caused an increase in PPO activity of roots whereas it remained unaffected in the shoots.

\section{Effect of FA pre-treatment on non-enzymatic antioxidants}

In comparison to water-primed seedlings, $100 \mathrm{ppm}$ of FA increased total phenolic content of wheat seedlings by more than $50 \%$. Ferulic acid treated wheat seedlings showed an increase in ascorbate content of roots by about 2 -fold in roots and 1.12-fold in shoots. In comparison to water-primed seedlings, proline content remained unaffected in wheat seedlings pre-treated with 100 ppm of FA.

\section{Discussion}

In comparison to hydro-primed seedlings, pre-treatment with 100 and $150 \mathrm{ppm}$ of FA promoted seedling growth of wheat accompanied by an increase in fresh weights of shoots but their dry weights remained unaffected. Azooz (2009) positively correlated the increased water contents with ameliorative and growth promoting effects of phenolics. Increase in seedling growth due to exogenous SA was correlated with enhanced cell division within apical meristems (Shakirova et al. 2003). Higher concentration of FA (1000 ppm) inhibited seedling growth which was reflected by decrease in shoot length and biomass of roots and shoots (Table 1). These results are in harmony with Kovacik et al. (2009) who explained that high doses of SA led to sharp increase in soluble phenolics and lignin accumulation and thereby inhibition of growth. Based upon seedling growth, 100 ppm of FA was chosen for studying the effect of FA pre-treatment on antioxidant defense system.

\section{Influence of FA pre-treatment on antioxidative enzymes and $\mathrm{H}_{2} \mathrm{O}_{2}$ content}

Superoxide dismutase activity remained unaffected in FA-treated seedlings. In contrast to this, an increase in SOD activity has been reported in maize plants treated with cinnamic acid (Singh et al. 2013). Although cinnamic acid is not a phenolic acid but, both compounds share one group, i.e. propenoic acid. NADPH oxidases could play the role in conversion of superoxide anion into $\mathrm{H}_{2} \mathrm{O}_{2}$ ( $\mathrm{Li}$ et al. 2011). Increase in CAT activity of shoots after pre-treatment with lower concentration of FA is positively correlated with lesser endogenous $\mathrm{H}_{2} \mathrm{O}_{2}$ content. Similarly, Wan et al. (2014) observed an increase in CAT activity of cucumber seedlings pre-treated with caffeic acid under control as well as stressed conditions. Enhanced POX activity was also observed in maize plants pre-treated with SA (Saruhan et al. 2012). Scrutiny of present results showed that FA at lower concentrations induced CAT and POX activities which can protect plants by reducing toxic levels of $\mathrm{H}_{2} \mathrm{O}_{2}$ produced during cell metabolism. Ferulic acid upregulated APX activity in the roots and shoots of wheat seedlings. In contrast to this, Shi and Zhu (2008) found decreased APX activity on application of SA in cucumber plants. Glutathione reductase 
activity was downregulated in the shoots of FA-treated seedlings. Khan et al. (2010) also observed a decrease in GR activity of mungbean seedlings treated with $138 \mathrm{ppm}$ of SA. Increase in CAT, POX and APX activities was positively correlated with decrease in $\mathrm{H}_{2} \mathrm{O}_{2}$ content of FA-treated seedlings. It was also documented earlier that $\mathrm{H}_{2} \mathrm{O}_{2}$ content of eggplants decreased significantly on treatment with SA (Chen et al. 2011).

Pre-treatment with 100 ppm of FA increased PPO activity of roots. Polyphenol oxidase catalyses the oxidation of polyphenols into quinones using molecular oxygen as an electron acceptor. War et al. (2011) also reported upregulation of PPO activity in groundnut seedlings treated with SA. Wheat seedlings primed with $100 \mathrm{ppm}$ of FA showed increased total phenolic content. Singh et al. (2010) reported an increase in the levels of polyphenols after exogenous use of 50,100 and $150 \mathrm{ppm}$ of FA in pea leaves. Correlation between phenols and PPO activity revealed that enhanced phenolic content could be attributed to increased activity of phenylalanine-ammonia lyase (PAL) which catalyses the first committed step of Phenyl propanoid pathway.

Increase in ascorbate levels was also reported in SA-treated eggplants grown under under control and stressed conditions (Chen et al. 2011). Correlation between ascorbate content and APX activity suggests that FA induced increase in ascorbate content might be due to higher activities of enzymes involved in its biosynthesis. Wan et al. (2014) observed elevated levels of ascorbate content due to upregulation of monodehydroascorbate reductase (MDHAR) and dehydroascorbate reductase (DHAR) in cucumber seedlings pre-treated with caffeic acid. Proline content remained unaffected in wheat seedlings pretreated with 100 ppm of FA. Ghafiyehsanj et al. (2013) observed that 200 and 400 ppm of SA did not affect proline content of wheat under controlled conditions. However, in contrast to this Gautam and Singh (2009) found that application of SA decreased proline content in maize seedlings.

Pre-treatment with 100 ppm of FA promoted seedling growth of wheat by upregulating CAT, POX and APX activities, accompanied by decrease in $\mathrm{H}_{2} \mathrm{O}_{2}$ content. Although pretreatment with 100 ppm of FA caused downregulation of GR in the shoots and upregulation of PPO in the roots, but ascorbate and total phenolic contents increased significantly. This might be due to the activation of the enzymes involved in de novo synthesis of these compounds. A perusal of results showed that lower concentrations of FA can be used for pre-treatment of seeds to promote the antioxidant response so that plants can be protected against various upcoming environmental constraints. Therefore in addition to SA, FA could also act as a novel inducer of stress responsive genes.

\section{References}

Alexieva, V., Sergiev, I., Mapelli, S., Karanov, E. 2001. The effect of drought and ultraviolet radiation on growth and stress markers in pea and wheat. Plant Cell and Environ. 24:1337-1344.

Appu, M., Muthukrishnan, S. 2014. Foliar application of salicylic acid stimulates flowering and induce defense related proteins in finger millet plants. Universal J. Plant Sci. 2:14-18.

Azooz, M.M. 2009. Salt stress mitigation by seed priming with salicylic acid in two faba bean genotypes differing in salt tolerance. Intl. J. Agric. Biol. 11:343-350. 
Bates, L.S., Waldren, R.P., Teare, I.D. 1973. Rapid determination of free proline for water stress studies. Plant Soil. 39:205-207.

Chen, K., Arora, R. 2013. Priming memory invokes seed stress-tolerance. Environ. Exp. Bot. 94:33-45.

Chen, S., Zimei, L., Cui, J., Jiangang, D., Xia, X., Liu, D., Yu, J. 2011. Alleviation of chilling induced oxidative damage by salicylic acid pretreatment and related gene expression in eggplant seedlings. Plant Growth Regul. 65:101-108.

Chugh, V., Kaur, N., Gupta, A.K. 2011. Evaluation of oxidative stress tolerance in maize (Zea mays L.) seedlings in response to drought. Indian J. Biochem. Biophys. 48:47-53.

Devi, R., Kaur, N., Gupta, A.K. 2012. Potential of antioxidative enzymes in depicting drought tolerance of wheat (Triticum aestivum L.). Indian J. Biochem. Biophys. 49:257-265.

Foyer, C.H., Noctor, G. 2013. Redox signaling in plants. Antioxidants Redox Signal. 18:2087-2090.

Gautam, S., Singh, P.K. 2009. Salicylic acid-induced salinity tolerance in corn grown under NaCl stress. Acta. Physiol. Plant. 31:1185-1190.

Ghafiyehsanj, E., Dilmaghani, K., Shoar, H.H. 2013. The effects of salicylic acid on some of biochemical characteristics of wheat (Triticum aestivum L.) under salinity stress. Ann. Biol. Res. 4:242-248.

Guo, W., Beta, T. 2013. Phenolic acid composition and antioxidant potential of insoluble and soluble dietary fibre extracts derived from select whole-grain cereals. Food Res. Intl. 51:518-525.

Kang, G.Z., Li, G.Z., Liu, G.Q., Xu, W., Peng, X.Q., Wang, C.Y., Zhu, Y.Z., Guo, T.C. 2013. Exogenous salicylic acid enhances wheat drought tolerance by influence on the expression of genes related to ascorbateglutathione cycle. Biol. Plant. 57:718-724.

Khan, N.A., Syeed, S., Masood, A., Nazar, R., Iqbal, N. 2010. Application of salicylic acid increases contents of nutrients and antioxidative metabolism in mungbean and alleviates adverse effects of salinity stress. Intl. J. Plant Biol. 1:1-8.

Kovacik, J., Gros, J., Backor, M., Miroslav, S., Repcak, M. 2009. Salicylic acid-induced changes to growth and phenolic metabolism in Matricaria chamomilla plants. Plant Cell Rep. 28:135-143.

Li, Q., Yu, B., Gao, Y., Dai, A.H., Bai, J.G. 2011. Cinnamic acid pretreatment mitigates chilling stress of cucumber leaves through altering antioxidant enzyme activity. J. Plant Physiol. 168:927-934.

Lowry, O.H., Rosebrough, N.J., Frase, A.T., Randall, R.J. 1951. Protein measurement with folin phenol reagent. J. Biol. Chem. 193:265-275.

Saruhan, N., Saglam, A., Kadioglu, A. 2012. Salicylic acid pretreatment induces drought tolerance and delays leaf rolling by inducing antioxidant systems in maize genotypes. Acta Physiol. Plant. 34:97-106.

Shakirova, F.M., Sakhabutdinova, A.R., Bezrokuva, M.V., Fatkhutdinova, D.R. 2003. Changes in the hormonal status of wheat seedlings induced by salicylic acid and salinity. Plant Sci. 164:317-322.

Sharma, P., Jha, A.B., Dubey, R.S., Pessarakli, M. 2012. Reactive oxygen species, oxidative damage, and antioxidative defense mechanism in plants under stressful conditions. J. Bot. 2012.26 p. doi:10.1155/2012/217037.

Shi, Q., Zhu, Z. 2008. Effect of exogenous salicylic acid on manganese toxicity, element contents and antioxidative system in cucumber. Environ. Exp. Bot. 63:317-326.

Singh, D.P., Bahadur, A., Sarma, B.K., Maurya, S., Singh, H.B., Singh, U.P. 2010. Exogenous application of L-phenylalanine and ferulic acid enhance phenylalanine ammonia lyase activity and accumulation of phenolic acids in pea (Pisum sativum) to offer protection against Erysiphe pisi. Arch. Phytopatho. Plant Protect. 43:1454-1462.

Singh, P.K., Singh, R., Singh, S. 2013. Cinnamic acid induced changes in reactive oxygen species scavenging enzymes and protein profile in maize (Zea mays L.) plants grown under salt stress. Physiol. Mol. Biol. Plant. 19:53-59.

Srinivasan, M., Sudheer, A.R., Menon, V.P. 2007. Ferulic acid: Therapeutic potential through its antioxidant property. J. Clin. Biochem. Nutr. 40:92-100.

Swain, T., Hills, W.E. 1959. The phenolic constituents of Prunus domestica. I. The quantitative analysis of phenolic constituents. J. Sci. Food Agric. 10:63-68.

Teixeira, J., Gaspar, A., Garrido, E.M., Garrido, J., Borges, F. 2013. Hydroxycinnamic acid antioxidants: an electrochemical overview. Bio. Med. Res. Intl. 11 p. http://dx.doi.org/10.1155/2013/251754 
Wan, Y.Y., Chen, S.Y., Huang, Y.W., Li, X., Zhang, Y., Wang, X.J., Bar, J.G. 2014. Caffeic acid pre-treatment enhances dehydration tolerance in cucumber seedlings by increasing antioxidant enzyme activity and proline and soluble sugar contents. Sci. Hort. 173:54-64.

War, A.R., Lingathurai, S., Paulraj, M.G., War, M.Y., Ignacimuthu, S. 2011. Oxidative response of groundnut (Arachis hypogea) plants to salicylic acid, neem oil formulation and Aclypha fruitcosa leaf extract. Am. J. Plant Physiol. 6:209-219.

Zauberman, G., Ronen, R., Akerman, M., Wrksler, A., Rot, I., Fuchs, Y. 1991. Postharvest retention of the red color of litchi fruit pericarp. Sci. Hort. 47:89-97. 